\title{
Database Design on Background Software of Psychology Class
}

\author{
Shan $\mathrm{Hu}$ \\ School of Education and Sports, Bohai University, Jinzhou, 121013, China \\ guoxiaotian24@163.com
}

Keywords: mental health; network; database design; background software

\begin{abstract}
With the social development and progress, mental health education has become an irreplaceable part of our education in mental health education. To realize the psychological education truly, a system for students to study mental health knowledge is needed to develop in modern that network are in continuous evolution. Database design on background software of psychology class was carried on in the light of the problems of the existing background software of psychology class to provide support for the development of this software. This paper bases on requirement analysis to conduct conceptual structure design and logical structure design. In the phase of conceptual structure design, E-R diagram is used to represent relationships between entities; in the phase of logic structure design, using student information table to design table structure. The software based on this paper can help students quickly learn and master the mental health knowledge.
\end{abstract}

\section{Introduction}

In recent years, the issues of mental health education are gradually exposed. The number of students of suspension or withdrawal has increased year after year due to psychological problems cannot be normal learning and life. Students are facing the various aspects of the pressure, such as competitive pressure from social, living environment pressure, learning environment pressure, and even personal emotion pressure, and due to improper handle these pressures and lack of timely counseling, so college students that have mental health problems are more and more. In recent years, the phenomenon of violence, suicide or suspension, dropout caused by psychological problems is not uncommon. Figures provided by the Chinese center for disease control shows that 16\% 25.4\% of college students have anxiety, neurasthenia and depression and other psychological disorders [1]. Psychological problems of college students have been very serious and must arouse our attention. With the development of the times, especially with the wide application of computer network technology, computer network technology should be integrated into education, and this method can bring benefits not only for the development of computer technology, but also for the development of education. Background software of psychology class conforms to the development trend of discipline construction in network era [2]. Therefore, establishing psychological dredge software for students and teachers to communicate timely, and the software has a great help not only for college students, but also for the occurrence of various instability events. Background software of psychology class mainly completes the promotion and popularization for mental health knowledge, and it can help students to learn knowledge of conventional mental health, and students can communicate with teachers timely through this software to solve their problems. The software can embody, visualize abstract and difficult knowledge to help students master the mental health knowledge. 


\section{Requirement Analysis}

Requirement analysis is the starting point and basis of database design and it is also the basis for the design phase [3]. Requirement analysis is to solve the problem of the target system do. The task of software requirement analysis is to determine what work must be done by the system rather than to determine how the system complete the work, which is making complete, accurate, clear, specific requirements on the target system [4]. Requirement analysis is a key process of software engineering, and the mission in the phase of requirement analysis is to determine the functions of software system. With the rapid development of social industrialization, information technology, computer technology and application technology, a variety of software systems also will appear, software developers face application system complexity becomes more and more high, the scale is more and more big, support basic software system development, faced with the challenge of system complexity, diversity, uninterrupted and adaptability, and so on, a series of key issues. The importance and necessity requirement analysis also reflected, requirement analysis quality directly affect the progress of the entire software engineering and the final result. Bell and Thayer noted that "insufficient, contradictory and incomplete description of software requirements, is a key factor affecting the quality of software design." The purpose of this system is to design a platform which can help students learn mental health knowledge.

The portal can be divided into two parts, they are foreground and background. Foreground is mainly used by students, including hot articles, video movies, seminars and mood inn and so on; background is mainly used by teachers, teachers can maintain their and students' information and upload learning resources. In the foreground module, students can read hot articles and video movies online, or you can download them to your phone, iPAD or other mobile devices to read. For the lectures related psychology health, students can learn them at any time. The mood inn is a student self-organization communication platform which can publish mood, emotion articles, and love stories, touching stories, horror stories, jokes and phrases.

The mission of requirement analysis is to make clear requirements of users by detailed investigating real-world objects to be processed and fully understanding the work profile of original system, and then determine the functions of the new system based on it [5]. While the functional requirement is basic demand for software system, it is not the only demand. The following requirements are usually put forward for software system: functional requirement; performance requirement; reliability and availability requirement. Ample and accurate requirement analysis, as the foundation, determines the speed and quality of database building. The entire project could not achieve the desired effect if requirement analysis is done badly, or that the final product is not required by users.

\section{Conceptual Structure Design}

Conceptual structure design, or simple conceptual design for short, abstracts users' demands to information structure. The goal of conceptual design is to generate overall database concept structure reflected information needs of organization-wide, that is conceptual model [6]. Conceptual model can truly and fully reflect the real world, including relationships between things. Conceptual design usually has three categories: (1) top-down: defining the framework of the overall concept structure at first, and then gradually refining it. This method is suitable for those users are very clear the overall goal of the system, but do not quite understand the details; (2) bottom-up: first defining the conceptual structure of each topical application, and then integrating them together to get overall concept structure. The method is applicable to that users understand the specific business better, but not sure the overall situation; (3) gradual expansion: starting from the core requirements, defining the core conceptual structure and then expanding outward, gradually generating other concepts structure until the overall concept structure in the snowball way. This method is suitable for intermediate users those are not very clear both the specific business and the overall goal. Conceptual design stage is a critical stage of database design, and a conceptual model independent 
to specific DBMS is formed by synthesizing, inducing and abstracting users' needs based on requirement analysis [7]. Using E-R diagram to describe conceptual model, entity relationship approach abstracts specific data and the collection of entities is abstracted into entities' type, and using the type of relationships between entities to reflect the internal connections between the things in real world. According to requirement analysis, there is a relationship type in system: a student can browse a variety of content categories, a content category can be learned by more students; a teacher can manage multiple content categories, and one kind of content category may also be managed by more teachers. Conceptual model shows in Fig. 1.

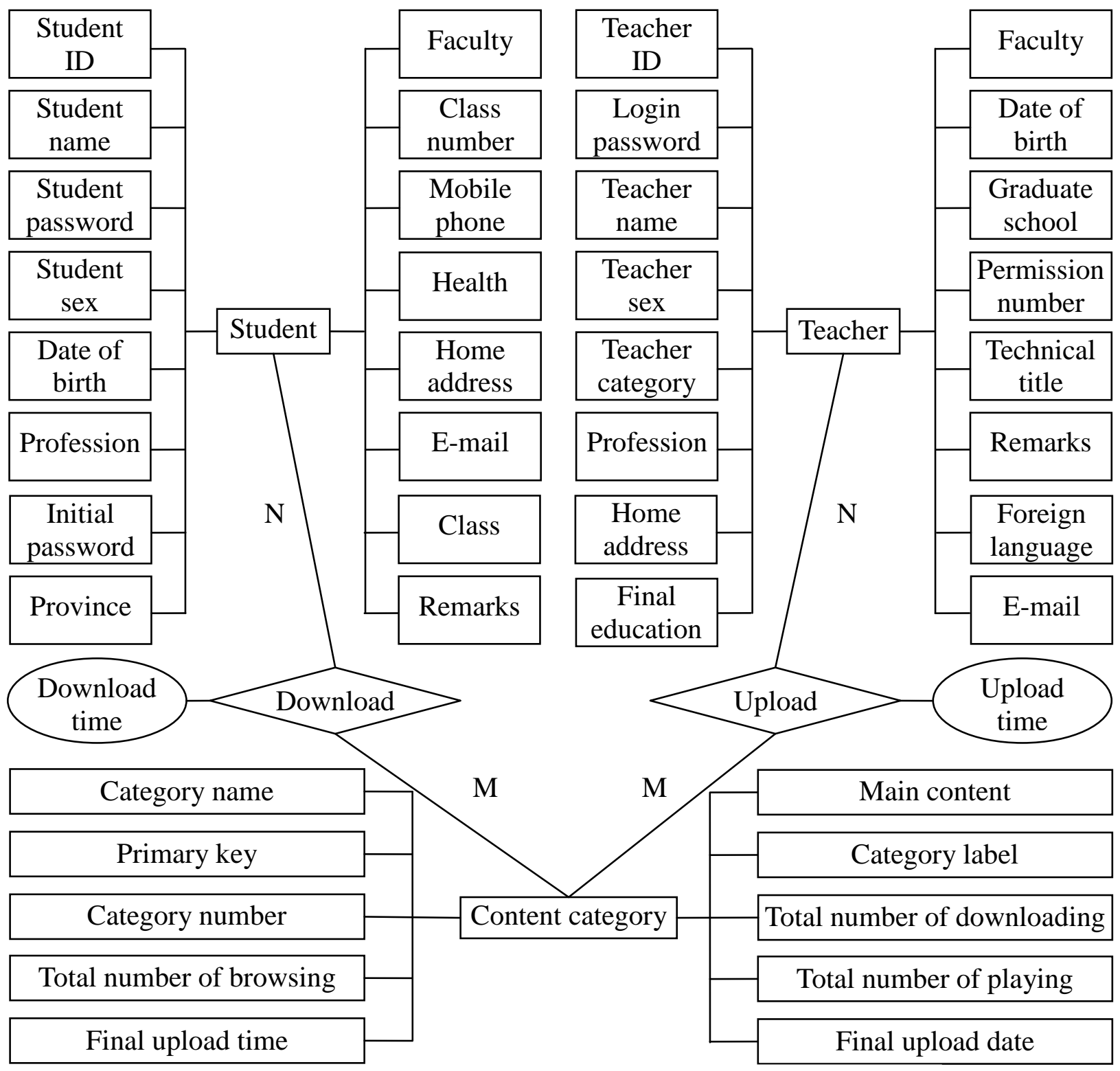

Fig. 1. E-R diagram of system

\section{Logical Structure Design}

Logical structure design, or simple logical design for short, is independent of any data model. In practice, the general database environment has been given. Because the database currently is the relational database, and therefore E-R diagram is needed to be converted to a relational model firstly, and then it should be converted to a data model supported by the particular DBMS according to the characteristics and limitations of specific DBMS, finally optimize it. Data structure in relational database is "table." Result of database logical design is not exclusive, preliminary data model should also be appropriately modified to adjust the structure of data model, so that the performance of database application system can be improved to enhance query speed. Given the 
different environments and tools provided by variety of DBMS, so the logical structure design is divided into the following steps: the conceptual model can be converted to relational model, network model, hierarchical model; the obtained relational model, network model, hierarchical model can be converted to data model supported by specific DBMS; adjusting and improving it based on requirement analysis and the features of specific DBMS [8]. The system includes student information table, teacher information table and content category table. Now take the student table as example, the logical structure design result of this system is as follows: the structure of student information table shows in Table 1.

Table 1. Student information table

\begin{tabular}{|c|c|c|c|}
\hline Number & Field name & Data type & Description \\
\hline 1 & Xsxh & Text & Student ID \\
\hline 2 & Xsxm & Text & Student name \\
\hline 3 & Xsxb & Text & Student sex \\
\hline 4 & Xsmm & Text & Student password \\
\hline 5 & Csrq & Datetime & Date of birth \\
\hline 6 & Scxy & Text & College \\
\hline 7 & Scxydm & Text & College code \\
\hline 8 & Sxzy & Text & Profession \\
\hline 9 & Sxzydm & Text & Profession code \\
\hline 10 & Ssbj & Text & Class \\
\hline 11 & Ssbjdm & Text & Class code \\
\hline 12 & Dzyx & Text & E-mail \\
\hline 13 & Yddh & Varchar & Mobile phone \\
\hline 14 & Szsf & Text & Province \\
\hline 15 & Szsfdm & Text & Province code \\
\hline 16 & Jtzz & Text & Home address \\
\hline 17 & Csmm & Text & Initial password \\
\hline 18 & Bjbh & Varchar & Class number \\
\hline 19 & Jkzk & Text & Health \\
\hline 20 & Bzsm & Text & Remarks \\
\hline
\end{tabular}

\section{Index Design}

Index provides a pointer to data values in the specified column of the storage table, and then you specify the sort order to sort these pointers [9]. The database uses the index to be very similar to the way you use index in a book: it searches the index to find a specific value, and then find the line that contains the value along the pointer. Efficient index design is extremely important to get good performance, try to experiment with different indexes. The index optimization wizard can analyze, query and recommend indexes should be created. The query optimizer in most cases reliably choose the most efficient index. The overall index design strategy should provide more opportunity of indexes choices for the query optimizer, and support them to make the right decision. This reduces analysis time in a variety of situations and achieve better performance.

Suggestions for creating an index are as follows:

(1) Will update as much as possible for multi-line query write a single statement, rather than using multiple queries to update the same row. Use only one statement, you can use the optimized 
index maintenance;

(2) Use the Index optimization wizard analyzes, query and obtain index recommendations;

(3) Use an integer key to the clustered index. In addition, the performance gain can be obtained by creating a clustered index on a unique column, a non empty column, or a IDENTITY column;

(4) Create a non-clustered index on all columns frequently used queries, you can maximize the use of covert inquiries;

(5) The time required to physically create the index depends largely on the disk subsystem;

(6) Check data distribution in the index column. Typically, the column that contains few unique values create indexes or perform a join on such a column, which will result in a long running query.

\section{Conclusion}

With the continuous progresses of society, psychological problems have been valued highly currently, especially the problems of college students. So the state has issued a number of important documents for the mental health education, reflecting the importance of mental health. The development of current mental health knowledge systems are not comprehensive, and cannot be truly student-centered. For the deficiencies and shortcomings of existing systems, this software designs and implements a psychology classroom system based on database technology, and the system gives full play to the advantages of the campus network to make up for deficiencies and shortcomings of traditional counseling methods, thereby to provide timely counseling services for college students. The software takes advantage of psychological service resources, and psychological problems of students will be solved effectively to protect their psychology develop to a more healthy direction. The application of background software of psychology class can maximum make up for the shortcomings of our psychology knowledge background, so that our students can have scientific guidance and basis when they have psychological problems [10]. Design of this system has great significance to improve the psychological knowledge for the college students.

\section{Acknowledgement}

This work is supported by "12th Five Year Plan" of educational science planning project of Liaoning province: Special subject research base for 2015 (JG15ZXY04). Subject name: Current situation investigation and countermeasure research of college students' mental health education for Liaoning province.

\section{References}

[1] J. L. Li, "Analysis of Problems and its Influencing Factors of the College Students' Psychological Health," Reform and Openning, vol. 25, no. 8, pp. 124-125, 2010.

[2] Q. L. Jiang, "Construction of University Network for Mental Health Education System," Doctor's degree of Central South University, 2012.

[3] J. M. Liu, "Student Physical Fitness Test System Database Design," Computer Knowledge and Technology, vol. 10, no. 20, pp. 4636-4639, 2014.

[4] Y. H. Meng, "Discuss on Requirement Analysis of Software Development Process," Science and Technology Information, vol. 26, no. 11, pp. 435-436, 2009.

[5] Baidu Encyclopedia, "Requirement Analysis," http://baike.baidu.com/view/43.htm, 2014-9-17.

[6] L. Zhang, L. Ma, "Database Design," Journal of Anyang Institute of Technology, vol. 6, no. 4, pp. 76-79, 2007.

[7] Douding Network, "Database Design," http://www.docin.com/p-552155016.html, 2014-9-18. 
[8] Z. Y. Wang, N. Chen, "Enterprise Database Design Based on Information Technology," Commercial Times, vol. 29, no. 5, pp. 67-68, 2010.

[9]Baidu Encyclopedia, "Database terminology: Index," http://baike.baidu.com/link?url=Kbn_2OyrzmL_78ndm9QfoZbQL6XxZhg59dbiY8byMkdlC6 pQBGTVCRwWm60XCIQ_NdBJ-awC1-OmydG3u0EJ8jPVi7p57zo8Lz3Zy77, 2015-7-20.

[10] M. Y. Li, "The Use of Psychological Evaluation Software to Carry Out Psychological Counseling," Today's Massmedia, vol. 19, no. 10, pp. 156-157, 2010. 